\title{
Quantization of Periodic Motions on Compact Surfaces of Constant Negative Curvature in a Magnetic Field
}

\author{
J. Brüning ${ }^{1}$, R. V. Nekrasov ${ }^{2}$, and A. I. Shafarevich ${ }^{3}$ \\ ${ }^{1}$ Humboldt-Universität zu Berlin \\ ${ }^{2}$ Institute for Problems in Mechanics, Russian Academy of Sciences \\ ${ }^{3}$ Moscow State University \\ Received May 17, 2006; in final form, June 28, 2006
}

\begin{abstract}
We use the semiclassical approach to study the spectral problem for the Schrödinger operator of a charged particle confined to a two-dimensional compact surface of constant negative curvature. We classify modes of classical motion in the integrable domain $E<E_{\text {cr }}$ and obtain a classification of semiclassical solutions as a consequence. We construct a spectral series (spectrum part approximated by semiclassical eigenvalues) corresponding to energies not exceeding the threshold value $E_{\mathrm{cr}}$; the degeneration multiplicity is computed for each eigenvalue.
\end{abstract}

DOI: $10.1134 / \mathrm{S} 0001434607010038$

Key words: Schrödinger equation, eigenvalue asymptotics, semiclassical approximation, confined classical motion, surface of negative curvature, symplectic structure.

\section{INTRODUCTION}

The motion of a charged particle in a magnetic field on the hyperbolic plane $L$ satisfies the standard Hamiltonian system

$$
\begin{aligned}
\dot{x}^{i} & =\frac{\partial H}{\partial p_{i}}, \quad i=1,2, \quad x^{1}=x, \quad x^{2}=y, \quad p_{1}=p_{x}, \quad p_{2}=p_{y} . \\
\dot{p}_{i} & =-\frac{\partial H}{\partial x^{i}},
\end{aligned}
$$

The magnetic field is given by a closed 2 -form on the hyperbolic plane; we assume that it is a multiple (with coefficient $w$ ) of the volume form. Then the Hamiltonian in the upper half-plane model has the form

$$
H=\frac{y^{2}}{2}\left(p_{x}-A_{x}\right)^{2}+\frac{y^{2}}{2}\left(p_{y}-A_{y}\right)^{2}, \quad A_{x}=\frac{w}{y}, \quad A_{y}=0, \quad w>0 .
$$

Isometries of the hyperbolic plane take trajectories to trajectories, and so the motion can be "factorized," i.e., considered on an arbitrary quotient surface $M$ of $L$ by a freely ${ }^{1}$ acting subgroup $\Gamma$ of the isometry group. We consider only compact orientable surfaces $M$, i.e., surfaces of constant negative curvature $K=-1$; the genus $g$ of such a surface is greater than 1 .

The motion in the corresponding phase space is determined by the Hamiltonian system with Hamiltonian $H_{M}=p^{2} / 2$ on the cotangent bundle $T^{*} M$ equipped with the symplectic structure [1]

$$
\Omega=d p_{i} \wedge d x^{i}+w \pi^{*} d S,
$$

where $d S$ is the area form on $M$ and $\pi$ is the natural projection.

\footnotetext{
${ }^{1}$ That is, without fixed points.
} 
The quantum system is given by the magnetic Schrödinger operator. On the hyperbolic plane, it is defined on functions and has the form

$$
\widehat{H}=-\frac{y^{2}}{2}\left\{\left(i h \frac{\partial}{\partial x}+\frac{w}{y}\right)^{2}+h^{2} \frac{\partial^{2}}{\partial x^{2}}\right\}: L_{2}(L) \rightarrow L_{2}(L) ;
$$

here $h$ is a parameter arising in the construction of the semiclassical approximation below.

The "quotient" quantum system on $M$ is determined by an operator acting on sections of a $U(1)$ bundle over $M$ with connection form $\alpha$ whose curvature is equal to $i w d S$. There exists a connection with this property, provided that the total magnetic flux through $M$ is an integer [2],

$$
n=\frac{w(2 g-2)}{h} \in \mathbb{Z}
$$

the number $n$ determines the first Chern class of the chosen bundle. The bundle also depends on $2 g$ parameters $\lambda_{i}$ in $H^{1}(M, U(1)) \simeq U^{2 g}(1)$, known as Aharonov-Bohm vortices (e.g., see [2]), and to each bundle there corresponds its own connection $\alpha$; therefore, associated with the surface $M$ is the family of operators $\widehat{H}_{M}=h^{2} \widehat{H}_{M, n}\left(\lambda_{i}\right)$, each of which acts on $L_{2}$-sections of the respective bundle. Sufficiently smooth connected domains on $L$ are coordinate neighborhoods on $M$ in which the operator $\widehat{H}_{M}$ corresponding to the connection $\alpha=i \mathbf{A}$ with potential $\mathbf{A}$ chosen in (1) has the form (2). Since the spectral series in question depend on the magnetic field alone [3], we shall simplify the computations by using different connections and omit the parameters $\lambda_{i}$ in the operators to make notation shorter.

The motion of a charged particle on the hyperbolic plane coincides with the motion along curves of constant curvature (e.g., see [4]), which are described in [5]. The problem on the classical motion on a closed orientable surface $M$ of constant negative curvature in the magnetic field specified by the volume form was studied in [1]. In particular, it was proved there that the system is integrable for $E<E_{\text {cr }}=w^{2} / 2$.

The spectrum, resolvent, and eigenfunctions of the operator $\widehat{H}$ on the hyperbolic plane were studied in [4] and [6]. The paper [3] gives exact formulas for series of eigenvalues of $\widehat{H}_{M}$ satisfying the condition $E<E_{\text {cr }}$.

The main result of the present paper is the construction of a semiclassical approximation describing the spectrum and the degeneration of the spectrum of the quantum Hamiltonian $\widehat{H}_{M}$ (in the integrable domain $E<E_{\mathrm{cr}}$ ). The corresponding formulas can be useful in the classification of spectral series and states of the operator $\widehat{H}_{M, n}$ as well as in the study of the perturbed problem (say, with a small electric potential).

\section{SEMICLASSICAL APPROXIMATION}

To a set of invariant isotropic submanifolds of a Hamiltonian system, one can assign semiclassical solutions; the relevant procedure is given by Maslov's theory of canonical operator and complex germ [7]-[9].

\subsection{Geometry of the Foliation of the Phase Space into Trajectories and Invariant Submanifolds}

As described in [1], all trajectories in $T^{*} M$ are closed for $0<E<E_{\mathrm{cr}}$, and their natural projections into $M$ have constant geodesic curvature equal to $k_{g}=w / \sqrt{2 E}$. Hence the system is not only integrable for $0 \leq E<E_{\text {cr }}$ (because, along with $H_{M}$, there exists an additional integral of motion for these $E$ ) but also degenerate.

For an additional integral $F: T^{*} M \rightarrow \mathbb{R}$ one can take a function depending only on the trajectory center [1] and hence specified by an arbitrary function $F_{0}: M \rightarrow \mathbb{R}$; we assume that $F_{0}$ is an analytic Morse function.

Each level set $H_{M}=E, F=f$ is an invariant set of the Hamiltonian flow on $T^{*} M$, and its connected components (in general position) are Liouville tori. Thus, the neighborhood

$$
U^{*}(M)=\left\{(\mathbf{x}, \mathbf{p}) \in T^{*} M: \frac{\mathbf{p}^{2}}{2} \leq E_{\mathrm{cr}} \Longleftrightarrow p \equiv|\mathbf{p}|<w\right\}
$$

MATHEMATICAL NOTES Vol.81 No. 12007 


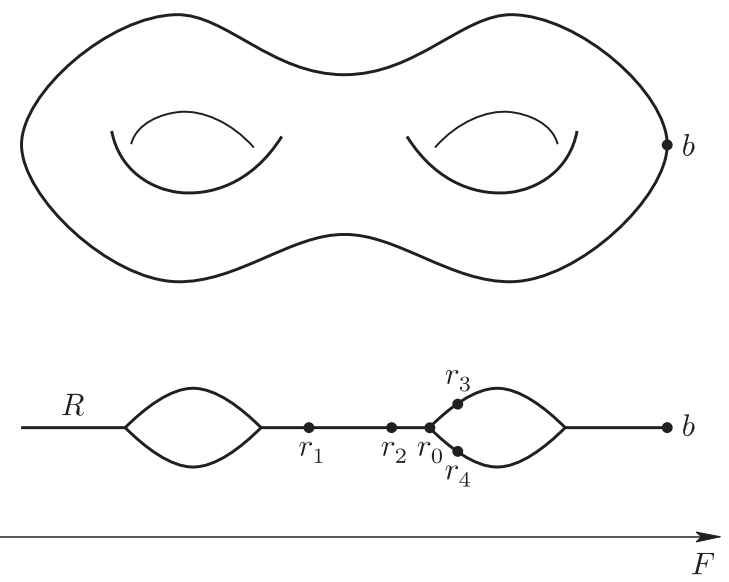

Figure: A surface of genus $g=2$ and the Reeb graph $R$ of the function $F$.

of the subset $M=\left\{(\mathbf{x}, \mathbf{p}) \in T^{*} M: p=0\right\}$ is foliated into invariant leaves. Let us show how $U^{*}(M)$ is foliated into such tori as well as singular leaves (equilibrium points, and bifurcations of Liouville tori). Owing to degeneration, this foliation depends on the choice of the additional integral $F$.

The surface $M$, i.e., the set of equilibria $(E=0)$, is foliated into closed curves and vertices, the latter being points of local maximum or minimum of the function $F_{0}=\left.F\right|_{E=0}$. The set of leaves can be visualized (e.g., see the example in the figure on the Reeb graph $R$ of the function $F_{0}$ (e.g., see [10]). Each point of the graph corresponds to a connected component of a level set; more precisely, the interior points of the edges correspond to closed curves without self-intersections (topological circles), and vertices correspond to isolated points or separatrix curves.

For $0<E<E_{\mathrm{cr}}$, the $(E, f)$-level set is the union of trajectories whose projections into $M$ are circles (in the constant curvature metric) of radius $\sqrt{2 E} / w$ centered on the $f$-level line, and each of its connected components is a union of trajectories centered on a connected component of the $f$-level line. If the $f$-component is a circle, then the $(E, f)$-component is a Liouville torus; an isolated point gives rise to a single circle (a "degenerate torus"), and a separatrix curve to a singular component (a separatrix, where a 2 -to- 1 or 1 -to-2 bifurcation of tori occurs ). Thus, the same $R$ specifies the foliation of any energy level $E, 0<E<E_{\mathrm{cr}}$, and the topology of the entire foliation $U^{*}(M)$ is specified by $\left[0, E_{\mathrm{cr}}\right) \times R$. Hence all connected components $\Lambda$ (including Liouville tori) of this foliation are parametrized by the pair $(E, r)$, where $E \in\left[0, E_{\mathrm{cr}}\right)$ and $r \in R$.

Finally, let us describe two action variables $I_{1}$ and $I_{2}$ for the Liouville tori of this foliation,

$$
I_{1}=I_{1}(E, r)=I_{1}(E), \quad I_{2}=I_{2}(E, r), \quad r \in R, \quad E \in\left[0, E_{\mathrm{cr}}\right) .
$$

To this end, from $M$ we delete the point $b$ of global maximum of $F$; then one can introduce a potential $\mathbf{A}$ on $M_{b}=M \backslash b$ and the 1 -form $\alpha=p_{i} d x^{i}+\mathbf{A}, d \alpha=\Omega$, on $T^{*} M_{b}$.

On each Liouville torus, we pick a pair of basis cycles consisting of an arbitrary trajectory $l_{0}$ oriented by the direction of motion and an arbitrary cycle $c$ that does not meet the boundaries of the natural projection of the Liouville torus (the cycle of singularities) and is oriented by its projection. (The sense of the projection supplemented by the gradient of $F$ should give a positive frame in $M$, i.e., a frame on which the volume form $d S$ is positive.) Then the expressions

$$
I_{1}=\frac{1}{2 \pi} \oint_{l_{0}} \alpha, \quad I_{2}=\frac{1}{2 \pi} \oint_{c} \alpha
$$

are well defined; the variable $I_{1}$ can additionally be defined in a similar way on all leaves, and $I_{2}$ can be defined on the leaves corresponding to interior points of edges of $R$.

Of the elementary properties of the action-angle variables (e.g., see [10]), we need the following.

Lemma 1. The variables $I_{1}$ and $I_{2}$ are independent of the choice of the cycles $l_{0}$ and $c$ and can be supplemented by angle variables $\varphi^{1}, \varphi^{2} \bmod 2 \pi$ on the Liouville torus, so that together they are Darboux coordinates,

$$
\Omega=d I_{i} \wedge d \varphi^{i}
$$


2.1.1. Computation of $I_{1}$ and $I_{2}$. The action $I_{1}$ is invariant with respect to the choice of the potential $\mathbf{A}$, because a trajectory bounds the two-dimensional disk of trajectories with lower energy and the same center and, by Stokes' theorem, the integration of $\alpha$ over the boundary can be replaced by the integration of $\Omega$ over the disk.

Obviously, each closed curve on $L$ covers a closed curve on $M$ under the projection $P: L \rightarrow M$, and each trajectory covers a trajectory. The converse, stronger assertion is also true.

Lemma 2. For each closed trajectory $l_{0}$ on $M$, the hyperbolic plane contains a closed trajectory (circle) $l$ that is projected one-to-one into $l_{0}$ under $P$.

Proof. Since the curvature of the covering curves is constant and less than 1 , it follows that all these curves are finite circles on $L$. Let a circle $l$ cover $l_{0}$ more than once; then $l$ contains two equivalent ${ }^{2}$ points $m_{2}$ and $m_{1}$ such that $m_{2}=g m_{1}$ for some $g \in \Gamma$. A sufficiently small arc in a neighborhood of $m_{1}$ is taken to an arc in a neighborhood of $m_{2}$, and hence the proper transformation $g \in \Gamma$ takes the entire circle $l$ into itself. Consequently, the center $m_{0}$ of the circle is a fixed point of $g$. This is a contradiction, because the covering $P: L \rightarrow M$ is defined by a discrete group $\Gamma$ acting freely (without fixed points).

Corollary. For each two-dimensional surface $\Sigma_{0} \subset T^{*} M$ bounded by the trajectory $l_{0}$, there exists a two-dimensional surface $\Sigma \subset T^{*} L$ projected one-to-one into $\Sigma_{0}$ under $P$.

By this corollary, every disk can be "lifted" from $T^{*} M$ to $T^{*} L$. Since the symplectic structure is preserved by the covering as well as, conversely, by the "lifting," one can integrate the form $\Omega$ over the corresponding disk in $T^{*} L$. We use Stokes' theorem once more (to simplify the computations) and evaluate $I_{1}$ on the trajectories already lifted to $T^{*} L$ (cf. [4]).

Consider an arbitrary circle

$$
\begin{aligned}
& x(\varphi)=x_{0}-R \sin \varphi \\
& y(\varphi)=y_{0}+R \cos \varphi
\end{aligned}
$$

on $L$; the corresponding trajectory is

$$
\begin{gathered}
x(\varphi)=x_{0}-R \sin \varphi \\
y(\varphi)=y_{0}+R \cos \varphi \\
p_{x}=-\frac{\sqrt{2 E}}{y_{0}}+\frac{w}{y_{0}}=\text { const, } \quad p_{y}(\varphi)=\frac{\sqrt{2 E}}{y} \sin \varphi .
\end{gathered}
$$

Hence

$$
\begin{aligned}
I_{1} & =\frac{1}{2 \pi} \int_{0}^{2 \pi}\left\{p_{x} R \cos \varphi+\frac{\sqrt{2 E} \sin \varphi}{y} R \sin \varphi\right\} d \varphi \\
& =\frac{R \sqrt{2 E}}{2 \pi y_{0}} \int_{0}^{2 \pi} \frac{\sin ^{2} \varphi}{1+\left(R / y_{0}\right) \cos \varphi} d \varphi=\sqrt{2 E}\left(\frac{y_{0}}{R}-\sqrt{\frac{y_{0}}{R}-1}\right) .
\end{aligned}
$$

It is well known that

$$
\frac{R}{y_{0}}=\frac{1}{k_{g}}=\frac{\sqrt{2 E}}{w},
$$

and hence we eventually obtain

$$
I_{1}(E)=w-\sqrt{w^{2}-2 E}
$$

\footnotetext{
${ }^{2}$ In the sense of the covering.

MATHEMATICAL NOTES Vol.81 No. 12007
} 
Lemma 3. For E fixed, the variable $I_{2}$ increases along the edges of the Reeb graph together with $F$,

$$
r_{1}, r_{2} \in R, \quad r_{2} \sim r_{1}, \quad F\left(r_{2}\right)>F\left(r_{1}\right) \Longrightarrow I_{2}\left(E, r_{2}\right)-I_{2}\left(E, r_{1}\right)>0
$$

and experiences jumps at the branching vertices $r_{0}$ of $R$. These jumps satisfy Kirchhoff's law: the sum of right limits of $I_{2}$ on incoming edges (with lower value of $F$ ) is equal to the sum of left limits on the outgoing edges (with larger value of $F$ ). For example, if the $r_{i}, i=2,3,4$, are arranged as shown in the figure, then

$$
I_{2}\left(E, r_{3}\right)+I_{2}\left(E, r_{4}\right)-I_{2}\left(E, r_{2}\right) \rightarrow 0 \quad \text { as } \quad F\left(r_{i}\right) \rightarrow F\left(r_{0}\right) .
$$

The total variation of $I_{2}$ over all edges is equal to

$$
\Delta I_{2}(E)=\left(w-I_{1}(E)\right)(2 g-2) .
$$

Proof. It is easier to see this for the zero energy level $E=p=0$. Then one can assume that $\alpha=\mathbf{A}$ and $\Omega=w d S$ are forms on $M$ and compute the difference in the values of $I_{2}$ for two level lines $c_{1}$ and $c_{2}$ ("degenerate tori") of the function $F\left(F\left(c_{1}\right)<F\left(c_{2}\right)\right)$ on a common edge of $R$ by Stokes' theorem,

$$
I_{2}\left(0, r_{2}\right)-I_{2}\left(0, r_{1}\right)=\frac{1}{2 \pi} \iint_{\vartheta} w d S=\frac{w S(\vartheta)}{2 \pi}>0,
$$

where $\vartheta$ is the cylinder on $M$ bounded by the cycles $c_{1}$ and $c_{2}$ and $S$ is the area measure. To prove "Kirchhoff's law," one considers, in a similar way, cycles on all edges adjacent to a given vertex, uses Stokes' theorem, and lets the area of the surface on $M$ tend to zero by shrinking to the vertex the cycles bounding the surface. Finally, the maximum value $w S(M) /(2 \pi)$ (which is equal to $w(2 g-2)$ by GaussBonnet) can be obtained from the first two assertions and, again, Stokes' theorem, applied this time to the entire surface $M$.

Now let us describe how to compute the difference in $I_{2}$ for two tori $\Delta\left(E, r_{1}\right)$ and $\Delta\left(E, r_{2}\right)$ that have a same energy $E>0$ and correspond to a same edge.

To this end, we consider not only the energy level but also smaller values. Thus, in the phase space, we have a four-dimensional region $Q$ consisting of tori between $c_{1}, c_{2}$ with various energy $E$ from 0 to $p^{2} / 2$. The region $Q$ is bounded by the maximum energy level surface and by two surfaces of the tori over $c_{1}$ and $c_{2}$. The integral of the phase volume form $\Omega \wedge \Omega$ over $Q$ is equal to the volume $V(p)=\pi p^{2} \Delta S$ of $Q$. But the phase volume of $Q$ can be computed by the formula (see (4))

$$
V(p)=4 \pi^{2} \iint_{Q} d I_{1} d I_{2},
$$

and the volume increment $d V=\pi \Delta S d p^{2}$ is equal to

$$
4 \pi^{2} \Delta I_{2}\left(E, r_{2}\right) d I_{1} .
$$

We find the increment of $I_{1}$ from (5) and finally obtain

$$
I_{2}\left(E, r_{2}\right)-I_{2}\left(E, r_{1}\right)=\sqrt{w^{2}-p^{2}} \frac{S(\vartheta)}{2 \pi}>0,
$$

which gives "Kirchhoff's law" and the maximum variation in $I_{2}$ for surfaces bounded by cycles on distinct edges.

\subsection{Quantization of the Symbol $H_{M}$}

Let us describe the relationship between the symbol $H_{M}$ given by the Hamiltonian of classical motion and the operator $h^{2} \widehat{H}_{M, n}, n \sim 1 / h$; we intend to construct an approximation in the spectral problem for the latter by using information about the classical motion.

By definition, to pseudodifferential operators in $R^{k}$ one can assign symbols, which are smooth functions in $R^{2 k}$. The inverse operation $H(x, p) \rightarrow \widehat{H}=H(x, \widehat{p})$ is known as quantization of symbols. There is a deeper semiclassical-approximation relationship between symbols and operators: solutions 
of the Schrödinger equation with a given operator are approximated by well-known WKB-Maslov constructions [8], [9] involving solutions of the Hamiltonian system with the corresponding symbol. Semiclassical approximation can readily be generalized to Schrödinger equations on Riemannian manifolds $M^{k}$ instead of $R^{k}$. Solutions of the Schrödinger equation are then constructed with the use of solutions of the Hamiltonian equations in $T^{*} M^{k}$ with some symbol. Although in this case there are already no unambiguous invariant procedures assigning an operator to a symbol, all choices of such a procedure are equivalent in the semiclassical sense: semiclassical solutions approximate exact solutions of the Schrödinger equations for various operators corresponding to the same symbol equally well.

In the more general case of operators acting on sections of bundles over the configuration space $M^{k}$, there is no assignment of this type at all. The most general quantization of a symbol $W$ on an arbitrary symplectic manifold [7] gives operators $\widetilde{W}$ with more complicated domains than sections of $U(1)$ bundles over $M^{k}$; the operators $\widetilde{W}$ are defined on the so-called wave packet sheaf $\Pi\left(T^{*} M^{k}\right)$ (see [7] for details).

Locally, in connected domains $D$ of the phase space, one can introduce Darboux coordinates $(\mathbf{x}, \mathbf{p}) \in$ $R_{x}^{k} \times R_{p}^{k}$. The restriction of the sheaf $\Pi\left(T^{*} M^{k}\right)$ to such coordinate neighborhoods gives so-called local sheaves over these neighborhoods, i.e., functions $\psi \in C_{0}\left(R_{x}^{k}\right)$ modulo $O\left(h^{\infty}\right)$ in $D$, i.e., cosets of functions, where two functions $\psi_{1}$ and $\psi_{2}$ are said to be equivalent if we have $\left(\psi_{1}, \widehat{\chi} \psi_{2}\right)=O\left(h^{\infty}\right)$ for all $\chi \in C_{0}(D)$. It is on these local sheaves that the operator $\widetilde{W}$ can be represented as a pseudodifferential operator with symbol $W(\mathbf{x}, \mathbf{p})$.

The following conditions are necessary for the existence of the sheaf $\boldsymbol{\Pi}\left(T^{*} M^{k}\right)$ (see [7]):

$$
\frac{1}{2 \pi h} \int_{\Sigma} \Omega=n \in \mathbb{Z} \quad \forall \Sigma: \quad \partial \Sigma=0 .
$$

In our case, it suffices to verify these conditions only for surfaces $\Sigma \subset M$, since on can smoothly shrink each surface to $p=0$. Then $M$ is the only nonzero surface without boundary, and condition (7) acquires the form (3).

The construction of such sheaves is ambiguous. Distinct nonequivalent sheaves $\Pi\left(T^{*} M\right)$ are parameterized by elements of the cohomology group $H^{1}\left(T^{*} M, U(1)\right) \simeq U^{2 g}(1)$, which is in our case isomorphic to the set $H^{1}(M, U(1))$ parametrizing the original operator. Thus, the quantized operator (which is an operator family with a small parameter $h$ ) is also defined only for a discrete set of values of the ratio $w / h$, and, up to the choice of parameters in $U^{2 g}(1)$, one has

$$
\widetilde{H}_{M}=\widetilde{H}_{M, n}: \Pi\left(T^{*} M\right) \rightarrow \Pi\left(T^{*} M\right), \quad n \sim \frac{1}{h}, \quad h \rightarrow 0 .
$$

Although the operators themselves do not coincide (they are defined on distinct spaces), $\widetilde{H}_{M, n}$ acts in "coordinate" representations in exactly the same way as $h^{2} \widehat{H}_{M, n}$. Indeed, by representing the action of the operator $\widetilde{H}_{M, n}$ in neighborhoods of the form $D=T^{*} U \subset T^{*} M$ with the Darboux coordinates

$$
\mathbf{p}^{\prime}=\mathbf{p}+\mathbf{A}, \quad \mathbf{x}^{\prime}=\mathbf{x}
$$

we obtain the operator $\mathbf{p}^{2} / 2$ acting on $C_{0}^{\infty}(U)$ as the differential operator with symbol $(\mathbf{p}-\mathbf{A})^{2} / 2$, exactly in the same way as this is defined in the Introduction for the action of the operator $\widehat{H}_{M, n}$ in $U$. Of course, the global sheaf is patched together by the same methods as bundles over $M$.

Thus, quantization of the symbol $H_{M}$ gives operators $\widetilde{H}_{M, n}$ whose action on sections on the sheaf $\Pi\left(T^{*} U\right)$ exactly coincides in "coordinate" representations with the action of $h^{2} \widehat{H}_{M, n}$. It follows that approximate solutions of the spectral problem for the operator $\widetilde{H}_{M, n}$ automatically give equally approximate solutions of the spectral problem for $h^{2} \widehat{H}_{M, n}$. The semiclassical approximation for $\widetilde{H}_{M}$ (just as for arbitrary $\widetilde{W}$ ) is known; it is based on the construction of Maslov's canonical operator. 


\subsection{Outline of the Semiclassical Approximation}

In the construction of asymptotic solutions on the basis of Liouville tori, one encounters yet another obstruction when trying to patch together the canonical operators locally defined on the Liouville tori in a semiclassically well-defined way. This is essentially the quantization of the Liouville tori themselves. For each value of the parameter $h$, this quantization singles out a set of Liouville tori, and the global canonical operators $K_{\Lambda}: C_{0}^{\infty}(\Lambda) \rightarrow \Pi\left(T^{*} M\right)$ defined for these tori explicitly specify asymptotic solutions $\Psi=$ $K_{\Lambda}[1], E=H_{M}(\Lambda)$.

Let a set of basis cycles $\Gamma_{i}, i=1,2, \ldots, 2 g$, in $H_{1}\left(T^{*} M\right)$ be fixed. Consider surfaces $\Sigma \subset T^{*} M$ such that the boundary of $\Sigma$ consists of a cycle on $\Lambda$ and, possibly, some set of cycles $\Gamma_{i}$,

$$
\Sigma: \quad \Sigma \backslash \Lambda=\bigcup_{i \in I} \Gamma_{i}, \quad I \subset\{1,2, \ldots, 2 g\} .
$$

The torus $\Lambda$ is quantized if

$$
\frac{1}{2 \pi h} \int_{\Sigma} \Omega=n+\frac{\operatorname{Ind} \Sigma}{4}
$$

for each surface of the form (8), where the index Ind $\Sigma$ is defined in [7]. We need only the additivity of the index (if we treat a surface as two surfaces glued together along some common boundary on $\Lambda$, then the sum of their indices is equal to the index of the original surface), the fact that the index of a closed surface is zero [7], and the invariance of the index under continuous deformations of the surface (which is obvious, since otherwise these conditions cannot hold, say, for neighboring surfaces).

The semiclassical results obtained below are justified by the assertion that the section $\Psi=K_{\Lambda}[\mathbf{1}]$ satisfies, modulo $O\left(h^{2}\right)$, the spectral equation for $\widetilde{H}_{M, n}$ with some fixed (by the choice of basis cycles) parameters in $H^{1}\left(T^{*} M, U(1)\right)$ (see. [7, Chap. IV, Theorem 3.2]).

\subsection{Asymptotic Spectrum. Degeneration}

Let us use the quantization rules (9) to compute the asymptotic spectrum (and its degeneration) for the operator $h^{2} \widehat{H}_{M, n}, n \sim 1 / h$.

Theorem. Let

$$
I_{1}=h\left(m+\frac{1}{2}\right), \quad I_{2}=h l+C, \quad m, l \in \mathbb{Z},
$$

where the constant $C$ depends only on the edge of the Reeb graph and on the choice of the cycles $\Gamma_{i}$ (and hence on the choice of parameter values in $H^{1}\left(T^{*} M, U(1)\right)$ for the operator $\left.\widetilde{H}_{M, n}\right)$. Then the Liouville torus $\Lambda=\Lambda\left(I_{1}, I_{2}\right)$ satisfies the quantization condition, and the section $\Psi=K_{\Lambda}[\mathbf{1}]$ satisfies the spectral equation for $\widetilde{H}_{M, n}$ modulo $O\left(h^{2}\right)$,

$$
\widetilde{H}_{M} \Psi=E \Psi+O\left(h^{2}\right)
$$

Proof. 1. Necessity. If the surface $\Sigma$ lies entirely in a subset of the phase space where the symplectic form $\Omega$ has a primitive, $\Omega=d \alpha$, then Stokes' theorem reduces the integral on the left-hand side in (9) to the form

$$
\frac{1}{2 \pi} \oint_{\partial \Sigma} \alpha
$$

and the quantization rules for a connection with unit Aharonov-Bohm vortex parameters should become the ordinary Bohr-Sommerfeld rules. In other words, the index on the left-hand side is equal to the Maslov index of the curve $\gamma=\partial \Sigma \cap \Lambda$, and hence the following assertion requires no further proof. 
Lemma 4. If the surface $\Sigma$ entirely lies in a subset of the phase space where the symplectic form $\Omega$ has a primitive, $\Omega=d \alpha$, then (9) is equivalent to the equation

$$
\frac{1}{2 \pi} \oint_{\gamma} \alpha=h\left(n+\frac{\text { Ind } \gamma}{4}\right)+C
$$

where

$$
C=-\frac{1}{2 \pi} \oint_{\partial \Sigma \backslash \gamma} \alpha
$$

For the subset on which $\Omega$ is exact we take the set $T^{*} M_{b}$ described in Sec. 2 .

Let us indicate surfaces bounded by the basis cycles of the torus $\Lambda$, satisfying the assumptions of Lemma 4, and such that Eqs. (11) acquire the form (10).

The first surface is either the disk already mentioned in the computation of $I_{1}$ or, if the projection of the disk into $M$ contains the point $b$, its complement in $\bar{\Sigma} \simeq M$. Since $\gamma=l_{0}$ is the only boundary of this surface, we obtain $C=0$, and the left-hand side of (11) coincides with the definition of $I_{1}$.

The second surface is a surface spanning the basis cycles $\Gamma_{i}$ and the cycle $\gamma=c$ and lying in $T^{*} M_{b}$. For this surface, the constant $C$ is nonzero in general.

2. Sufficiency. If a surface $\Sigma$ with boundary $\gamma$ on the torus can be smoothly deformed from $T^{*} M$ into $T^{*} M_{b}$ (for example, if $\Sigma \subset T^{*} M_{b}$ from the very beginning) and conditions (11) hold for all basis cycles on the torus, the conditions (11) also hold for $\gamma$, and hence $\Sigma$ satisfies condition (9) by Lemma 4 .

If $\Sigma$ cannot be deformed in such a way, then (provided that the basis cycles $\Gamma_{i}$ and the boundaries of $\Sigma$ on the torus $\Lambda$ itself are chosen so as to ensure that their projections into $M$ neither contain the point $b$ nor have self-intersections, which is easy to achieve), then there exists a closed surface $\bar{\Sigma}$ obtained from $\Sigma$ by attaching another surface $\Sigma_{0}$ such that $\Sigma_{0} \subset T^{*} M_{b}$. Conditions (9) for $\Sigma$ and $\Sigma_{0}$ are equivalent by (7), additivity of the index, and the fact that the index of $\bar{\Sigma}$ is zero. Again, condition (9) for $\Sigma_{0}$ is satisfied by Lemma 4 .

The second part of the assertion is a consequence of [7, Chap. IV, Theorem 3.2].

Corollary. The number E approximates a spectral point of the operator $\widetilde{H}_{M, n}$ and hence of the desired operator $h^{2} \widehat{H}_{M, n}$,

$$
\operatorname{dist}\left(E, \operatorname{Spec}\left(h^{2} \widehat{H}_{M, n}\right)\right) \leq O\left(h^{2}\right) .
$$

From (10) and (5), up to the constant correction term $R=-h^{2} / 8$, we obtain the known exact formula [3] for lower energy levels $\left(E<E_{\mathrm{cr}}\right)$.

Proposition 1. One has

$$
E_{m}=h w\left(m+\frac{1}{2}\right)-\frac{h^{2}}{2}\left(m+\frac{1}{2}\right)^{2}, \quad m+\frac{1}{2} \leq \frac{w}{h} .
$$

The degeneration with respect to the second quantum number $l$ can be computed separately for each edge of the Reeb graph $R$, and then one takes the sum.

Proposition 2. The semiclassical degeneration (i.e., the number of states described by the canonical operator) of the mth energy level is equal to

$$
(2 g-2)\left(n-m-\frac{1}{2}\right)
$$

Proof. By taking different connections (i.e., the terms $C$ in (11)) for different $h$, one can always ensure that the quantized values of the variable $I_{2}$ do not arrive at any vertices of the Reeb graph. Then, by Lemma 3, the total number of quantized states is

$$
\frac{\Delta I_{2}}{2 \pi h}=(2 g-2)\left(n-m-\frac{1}{2}\right),
$$

as desired. 
From the exact degeneration [3]

$$
\operatorname{dim} \operatorname{Ker}\left(h^{2} \widehat{H}_{M n}-E_{m}\right)=(2 g-2)\left(n-m-\frac{1}{2}\right),
$$

we see that the states described by the canonical operator also form a complete set.

\section{ACKNOWLEDGMENTS}

The authors wish to express gratitude to V. A. Geiler, S. Yu. Dobrokhotov, and A. V. Smirnov for helpful discussion.

This work was supported by the Russian Foundation for Basic Research under grant no. 0501-00968a and by the Deutsche Forschungsgemeinschaft and the Russian Academy of Sciences under research project no. 436 RUS 113/572.

\section{REFERENCES}

1. I. A. Taimanov, "On an example of a transition from chaos to integrability for magnetic geodesic flows," arXiv: math. DS/0312430v2 (2005).

2. J. Bolte and F. Steiner, "Flux quantization and quantum mechanics on Riemann surfaces in an external magnetic field," J. Phys. A 24 (16), 3817-3823 (1991).

3. E. V. Ferapontov and A. P. Veselov, "Integrable Schrödinger operators with magnetic fields: factorization method on curved surfaces," J. Math. Phys. 42 (2), 590-607 (2001).

4. A. Comtet, "On the Landau levels on the hyperbolic plane," Ann. Phys. 173 (1), 185-209 (1987).

5. G. A. Hedlund, "Fuchsian groups and transitive horocycles," Duke Math. J. 2 (3), 530-542 (1936).

6. A. Comtet and P. J. Houston, "Effective action on the hyperbolic plane in a constant external field," J. Math. Phys. 26 (1), 185-191 (1985).

7. M. V. Karasev and V. P. Maslov, Nonlinear Poisson Brackets. Geometry and Quantization [in Russian] (Nauka, Moscow, 1991).

8. V. P. Maslov, Complex WKB Method for Nonlinear Equations [in Russian] (Nauka, Moscow, 1977).

9. V. P. Maslov and M. V. Fedoryuk, Semiclassical Approximation for Equations of Quantum Mechanics [in Russian] (Nauka, Moscow, 1976).

10. A. V. Bolsinov and A. T. Fomenko, Introduction to the Topology of Integrable Hamiltonian Systems / in Russian) (Nauka, Moscow, 1997). 